\title{
A Study on Obsolescence Resolution Profiles
}

Francisco J. Romero Rojo ${ }^{1}$, Rajkumar Roy ${ }^{1}$, Essam Shehab ${ }^{1}$ and Kalyan Cheruvu ${ }^{1}$

${ }^{1}$ Decision Engineering Centre, Cranfield University, Cranfield, Bedfordshire, MK43 OAL, UK.

\{f.romerorojo;r.roy;e.shehab\}@cranfield.ac.uk

\section{Abstract}

This paper presents a study on assessing the probability of selecting a resolution approach to tackle an obsolescence issue. Firstly, the Delphi method was applied across an industry expert panel to derive a set of 15 Obsolescence Resolution Profiles (ORPs). The ORPs represent the probability of using each obsolescence resolution approach to tackle an obsolescence issue. Each ORP is characterised by the complexity level of the obsolete component and the level of proactiveness for obsolescence management, which have been identified as the two major factors that influence the probability of using each resolution approach. Secondly, the results were enhanced by means of the definitions refinement workshop. Finally, the ORPs were refined and validated by means of a workshop with experts based on the theoretical trends expected for each resolution probability. More than 40 experts in obsolescence from across the UK from defence, aerospace, railway and nuclear sectors have participated in the study.

\section{Keywords}

Obsolescence; Delphi method; EEE; Obsolescence Management; DMSMS 


\section{INTRODUCTION}

The rapid change in technology during the last few decades has exacerbated the obsolescence problem in the defence sector, especially for electronic, electromechanical and electrical (EEE) components, as their life-cycle is becoming increasingly shorter [1,2]. There are various resolution approaches that can be applied to tackle an obsolescence issue, which have been identified and defined as part of this research in collaboration with experts on obsolescence from across the UK defence sector.

Obsolescence is a major issue in long-life support systems and occurs when the spares of a component are no longer in stock or are no longer being produced by its suppliers or manufacturers $[3,4,5,6]$. This is having a serious impact on the sustainment of most platforms in the defence sector, as their life-cycles need to be extended for several decades $[7,8]$.

The United Kingdom Ministry of Defence (MoD) is promoting a move towards new business models that provide better value for money such as "Contracting for Availability" [9]. These new ways of contracting are transferring risks from the customer to the prime contractor, who is in a better position to manage them in the most cost-effective way [10]. Therefore, full obsolescence management is starting to be passed on to the supplier. This therefore makes it necessary, at the bidding stage, to estimate its cost so it can be included in the contract [11].

One of the main challenges of estimating the obsolescence cost is that, in most cases, the resolution approach to tackle a particular obsolescence issue cannot be specified in advance. Therefore, the aim of the study presented in this paper is to determine the probability of using each resolution approach to tackle an obsolescence issue, based on the experience of more than 40 industrial experts in obsolescence.

This study has five objectives. Firstly, to define the different resolution approaches that can be applied to tackle an obsolescence issue for an electronic component. Secondly, to define the complexity of electronic components and classify them accordingly. Thirdly, to categorise the obsolescence management strategies 
according to the level of proactiveness deployed. Fourthly, to estimate the probability of using each resolution approach to tackle an obsolescence issue, taking into account the complexity level of the electronic component and the obsolescence management level deployed. Finally, to compare the trends of those probabilities across different levels of complexity of the obsolete component and proactiveness level to manage obsolescence.

\section{RESEARCH METHODOLOGY}

Prior to starting the "Obsolescence Resolution Profiles Study", it was necessary to conduct a preliminary study to establish the basic concepts in the area of obsolescence management. Figure 1 shows the overall research methodology.

\subsection{Preliminary Study}

The differences between the terms "mitigation" and "resolution" have been clarified in collaboration with five experts from different organisations with a range of 5 to 12 years work experience in the field. It was identified that there was a lack of consistency across industry with the usage of these terms, with some individuals using them interchangeably. Therefore, it was necessary to define each one appropriately to provide a common understanding. Also, the possible resolution approaches that can be applied to tackle an obsolescence issue for an electronic component were identified and defined. This was based on literature, the Ministry of Defence 2004 Obsolescence Cost Metrics Study [12] and discussions with the five experts on obsolescence from industry.

The second step was the development of a classification of electronic components according to their level of "complexity". The concept of "complexity" can be regarded as tacit knowledge that obsolescence experts develop as they deal with obsolescence issues. In order to define explicitly this concept, the Critical Incident Technique [13] was followed during a 4-hour workshop with one obsolescence expert (7 years industry experience in the field). This methodology allowed capturing the logic and key parameters that define the complexity of an electronic component by comparing features of different components [14]. The outcomes of this session were refined and validated in collaboration with another obsolescence expert (10 years 
industry experience in the field). Subsequently, the concept has been presented at several Component Obsolescence Group (COG) quarterly meetings and conferences, where it was approved by the attendees (more than 70 obsolescence experts).

The next step was the classification of the obsolescence management strategies according to the level of proactiveness deployed. This classification was based on the information gathered through several semi-structured interviews with more than ten obsolescence experts from several defence organisations and an exhaustive literature review. It was refined and validated in collaboration with an obsolescence expert (7 years industry experience in the field). In the same manner as the complexity concept, this classification was presented at several COG quarterly meetings and conferences, where it was approved by the attendees (more than 70 obsolescence experts).

By means of the interactions with industry described hitherto, it was identified that the probability of using a resolution approach to tackle an obsolescence issue for an electronic component depends mainly on these two parameters: the level of complexity of the obsolete component and the level of proactiveness deployed to manage obsolescence. This novel finding provided the basis for the development of the Obsolescence Resolution Profiles (ORPs).

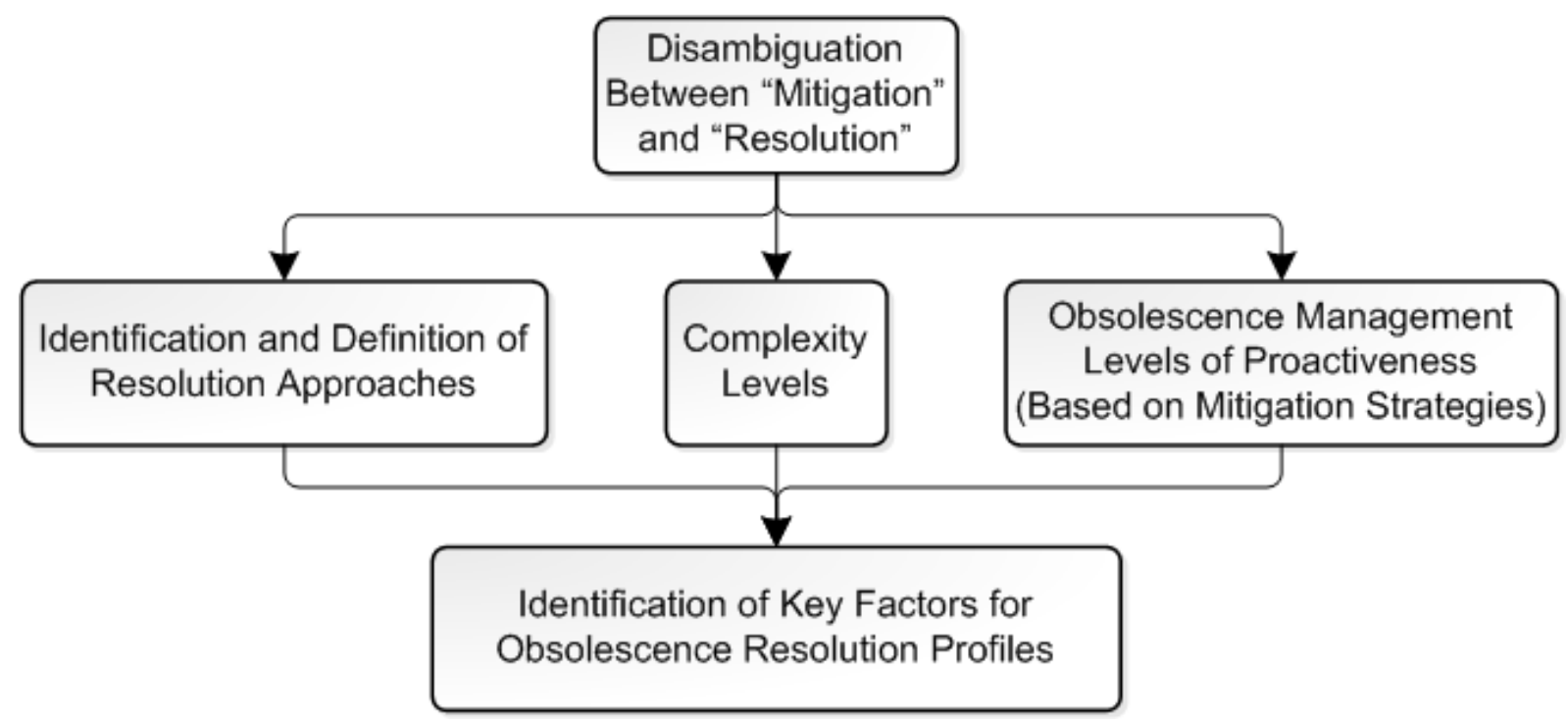

Figure 1 Preliminary Study Methodology

The Obsolescence Resolution Profiles Study is composed of three major phases, as shown in Figure 2. 


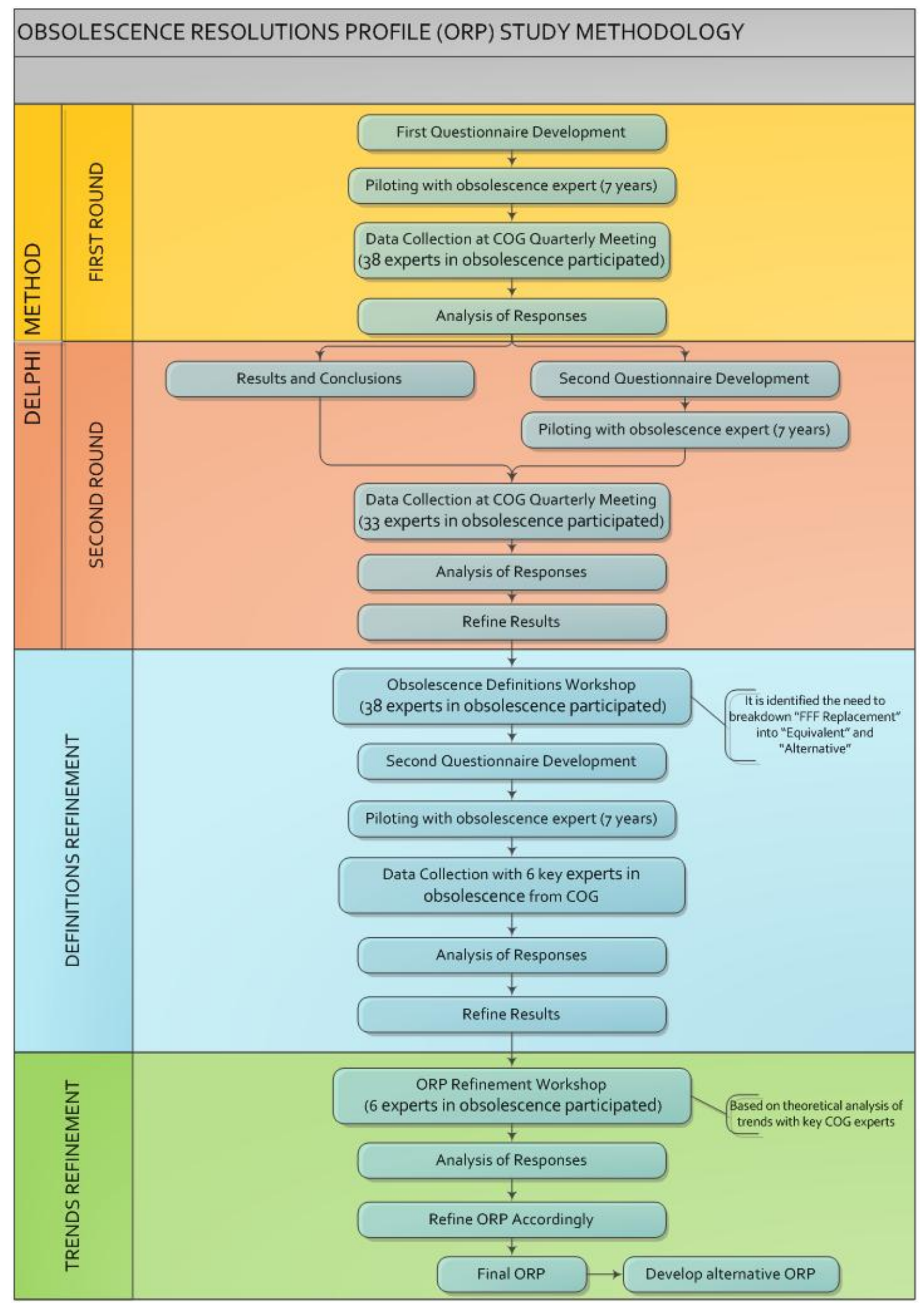

Figure 2 Obsolescence Resolution Profiles Study Methodology Phases 


\subsection{Phase 1: Delphi Method}

The results of the Preliminary Study and the membership of the Component Obsolescence Group (COG) set the appropriate circumstances for deploying the Delphi method to estimate the probability of using each resolution approach to tackle an obsolescence issue, taking into account the complexity level of the electronic component and the obsolescence management level. COG is a special interest group of like-minded professionals, from all levels of the supply chain, and across all industries and relevant Government agencies concerned with addressing and mitigating the effects of obsolescence [15]. The Delphi method is "an iterative process to collect and distil the anonymous judgments of experts using a series of data collection and analysis techniques interspersed with feedback" [16]. This research tool is particularly well suited to new research areas and exploratory studies such as the development of Obsolescence Resolution Profiles [17,18]. The key reason for using the Delphi method is the lack of appropriate historical data about the resolution approaches used to solve obsolescence issues, and thus expert judgment is required [19].

Firstly, a questionnaire was developed to be used in the first round with the COG panel (see Appendix 1). Prior to this, it was piloted with an obsolescence expert (7 years experience) to make sure it was clear, unambiguous and precise. In the questionnaire, the participant is initially requested to input the years of experience on obsolescence and the obsolescence management level that best represents the current practice of the company or project that they are involved in. Subsequently, the participant can assess the likelihood of having resolved an obsolescence issue following each of the given approaches for each complexity level of the component. Keeney and von Winterfeldt [20] support the use of probabilities to quantify expert judgements in examining complex technical and engineering problems. In the light of this, the score provided by each participant is based on an 11-point Likert Scale ranging from zero (never used) to 10 (certainly used), and during the analysis phase, these results are turned into percentages. A total of 38 experts in obsolescence participated in the first round and the responses were subsequently analysed. During this analysis, the mean and standard deviation of the responses were calculated, and 
a $95 \%$ confidence was considered in order to remove the outliers (the $5 \%$ of the responses, which were beyond the limits of this interval, were ignored).

The outcomes of this analysis were presented at a second round to 33 obsolescence experts, out of which 13 had also participated in the first round. They took the opportunity to discuss about the results and fill in a new questionnaire (see Appendix 2) either corroborating the results or correcting them. The participants were given the possibility to amend any percentages from any pie-chart, by writing the new figure next to the old one in the questionnaire, so that the rest of percentages would be adjusted during the analysis phase, keeping the same proportion. If the participant believes that no amendments are required for a pie-chart, they will circle the "OK" below it. A final analysis of these responses allowed refining the outcomes of the first round, providing as a result the obsolescence resolution profiles (ORPs).

The years of experience on obsolescence of the participants in the Delphi study are shown in Table 1.

Table 1 Years of Experience on Obsolescence of Participants in Delphi study

ROUND 1

\begin{tabular}{|c|c|}
\hline Years of Experience & Number of Participants \\
\hline Less than 5 years & 7 \\
\hline 5 years up to 9 years & 4 \\
\hline 10 years up to 19 years & 17 \\
\hline 20 years up to 29 years & 6 \\
\hline 30 years or more & 4 \\
\hline
\end{tabular}

ROUND 2

\begin{tabular}{|c|c|}
\hline Years of Experience & Number of Participants \\
\hline Less than 5 years & 5 \\
\hline 5 years up to 9 years & 4 \\
\hline 10 years up to 19 years & 17 \\
\hline 20 years up to 29 years & 4 \\
\hline 30 years or more & 3 \\
\hline
\end{tabular}

\subsection{Phase 2: Definitions Refinement}

A total of 38 obsolescence experts from different organisations participated in the "Obsolescence Definitions Workshop" in order to properly define the different resolution approaches and generate a common understanding of these terms across the UK defence sector. During Phase 1, one of the resolution approaches considered was the replacement of the obsolete component using another with the same Form, Fit and Function (FFF). However, the outcomes of the "Obsolescence Definitions Workshop" leaded to the decision of breaking down this approach into "Equivalent" and "Alternative". The distinction between them is that an equivalent component is functionally, parametrically and technically interchangeable with the obsolete one, 
while the performance of the alternative component may be different from that specified. Therefore, a subsequent study was carried out with six key obsolescence experts from five different organisations (their experience ranged from 11 to 40 years) to split the probability of using a FFF replacement into equivalent and alternative. A questionnaire was filled in by each expert, indicating the proportion in which the FFF replacement percentage needed to be broken down for each of the 15 ORPs. The responses collected were analysed, and the mean was applied to refine the ORPs resulting from the Delphi study.

\subsection{Phase 3: Trends Refinement - Validation}

The ORPs were analysed in terms of identifying the trends in probability of usage for each resolution approach across the different levels of complexity or levels of proactiveness to manage obsolescence. Those trends were presented at the "ORP Refinement Workshop", where six key experts on obsolescence from different organisations participated (their level of experience is detailed in Table 2). The objective was to validate the trends by checking their plausibility [21], making sure that the patterns and figures made sense. The experts discussed each trend, concluding whether it matched the anticipated trend based on their experience or not, and justifying it.

Table 2 Years of Experience on Obsolescence of Participants in Trends Refinement

\begin{tabular}{cc}
\hline Participant & Years of Experience \\
\hline Participant 1 & 12 \\
Participant 2 & 11 \\
Participant 3 & 5 \\
Participant 4 & 10 \\
Participant 5 & 3 \\
Participant 6 & 30 \\
\hline Average & 12 \\
\hline
\end{tabular}

Additionally, alternative obsolescence resolution profiles were derived to adapt them to the termination stage of the in-service phase (typically, the last five years of the project). During this phase, the likelihood of resolving obsolescence issues by means of cannibalisation and Last-time Buy (LTB) increases, while using equivalents and alternatives or doing redesigns or emulation become less likely. The assumption made for this adjustment is that the probability of using equivalents and alternatives, 
redesigns or emulation will reduce by $50 \%$. The increase in the probability of using cannibalisation and LTB will be proportional to their probabilities in the original obsolescence resolution profiles. In principle, the experts agreed that these adjustments are reasonable and make the alternative ORPs more suitable for the end of the in-service phase than the original ones.

\section{RESULTS}

The first outcome of this study was a set of definitions agreed across experts in the British defence sector. A distinction has been made between "mitigation strategies" and "resolution approaches". Mitigation strategies are those actions performed in order to reduce the risk or potential impact of obsolescence issues whereas resolution approaches are those actions carried out once an obsolescence issue arises and needs to be addressed.

\section{- Obsolescence Resolution Definitions}

The definitions for the different resolution approaches that can be applied to tackle an obsolescence issue for an electronic component are presented in Table 3. These resolutions can be grouped into four categories, as shown in Figure 3, which are: use of same obsolete component, use of a FFF replacement, emulation and redesign.

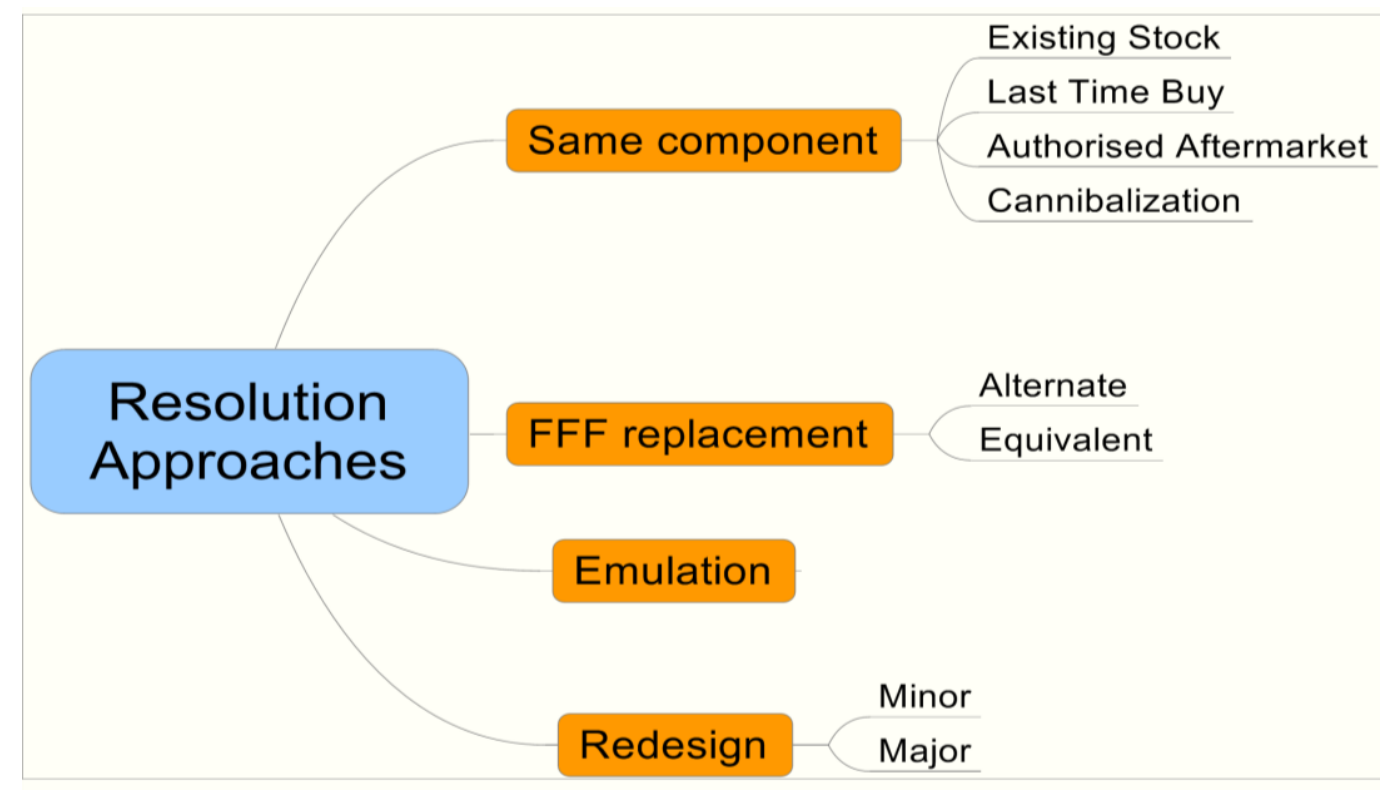

Figure 3 Obsolescence Resolution Approaches 
Table 3 Obsolescence Resolution Approaches Definitions

\begin{tabular}{|c|c|}
\hline Resolution Term & Definition \\
\hline Existing Stock & $\begin{array}{l}\text { Available item owned within the supply chain that can be } \\
\text { allocated to the project. }\end{array}$ \\
\hline Last Time Buy & $\begin{array}{l}\text { As a result of a product discontinuance notice, the } \\
\text { procurement of items sufficient to support the life cycle of the } \\
\text { project or until the next planned technology upgrade. }\end{array}$ \\
\hline Cannibalisation (Reclamation) & $\begin{array}{l}\text { The use of an item found in surplus equipment or equipment } \\
\text { beyond economical repair. }\end{array}$ \\
\hline Equivalent & $\begin{array}{l}\text { An item which is functionally, parametrically and technically } \\
\text { interchangeable (form, fit and function). }\end{array}$ \\
\hline Alternative (Alternate) & $\begin{array}{l}\text { An item whose performance may be different from that } \\
\text { specified for one or more reasons (e.g., quality or reliability } \\
\text { level, tolerance, parametric, temperature range). }\end{array}$ \\
\hline Authorised Aftermarket & $\begin{array}{l}\text { An item is available on the market but not from the original } \\
\text { manufacturer or supplier (typically finished goods provided by } \\
\text { licensed sources). Note that the components in this category } \\
\text { must have the same specifications as the original ones. }\end{array}$ \\
\hline Emulation & $\begin{array}{l}\text { A manufacturing process that produces a substitute form, fit } \\
\text { and function, and interface (F3I) item for the unobtainable } \\
\text { item. Microcircuit emulation can replicate with state-of-the art } \\
\text { devices that emulate the original and can be manufactured } \\
\text { and supplied on demand. }\end{array}$ \\
\hline Redesign & $\begin{array}{l}\text { An obsolete item is designed out of the system. Usually used } \\
\text { as a last resort because of the cost implications. Redesign } \\
\text { typically has the goal of enhancing system performance and } \\
\text { improving reliability and maintainability. The cost for redesign } \\
\text { can include engineering, programme management, } \\
\text { integration, qualification and testing. Redesign can be further } \\
\text { broken down into categories, e.g. minor (board re-layout) and } \\
\text { major (board replacement). }\end{array}$ \\
\hline
\end{tabular}

Due to the disambiguation between the terms "Emulation", "Minor Redesign" and "Major Redesign", defined in Table 3, it can be assumed that:

- The major redesign takes place at the subsystem/assembly level

- The emulation takes place at the assembly/board level

- The minor redesign takes place at the board/part level 
Considering the following levels in the system hierarchy:

○ Subsystem (e.g. radar, avionics)

- Assembly (e.g. display)

- Board (e.g. graphics card)

- Part (e.g. resistor)

\section{- EEE Component Complexity}

The second outcome of this study is the explanation of the "complexity" concept for electronic components, so they can be classified accordingly. The level of complexity will influence the probability of using each resolution approach to solve an obsolescence issue. Electronic components can be classified into three categories based on the complexity level: low, medium and high. Each category and its characteristics are shown as follows (Figure 4).

\section{High Complexity}

- Custom/Bespoke

- Single Source

- Small/Low reliable suppliers

- Cutting edge technology

- High cost

- No backwards compatible

- Low transparency in design

- Not easy to emulate

- Short life-cycles

- ๑.g. LCD Display and Microprocessor

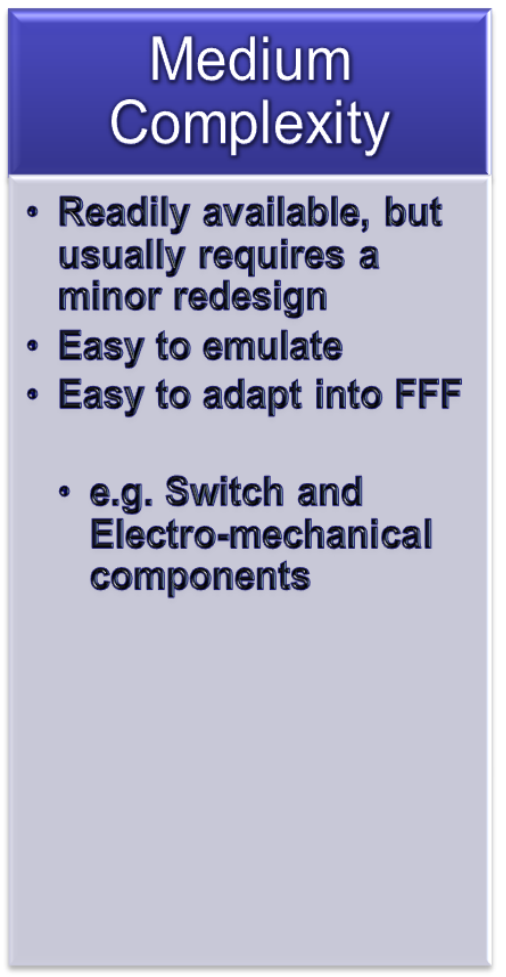

Figure 4 EEE Components Complexity Levels 
High-complexity components are characterised as bespoke, expensive, cutting-edge technology and no-backwards compatible. In general, this type of components is single-source supplied and the suppliers are usually small or low reliable. The lifecycle of these components is usually very short (around 1 or 2 years) and they are not easy to emulate. Examples of this type of component are LCD displays and microprocessors.

Medium-complexity components are usually readily available and easily adapted into FFF replacements. Furthermore, they are easy to emulate. Examples of this type of component are switches and electromechanical components.

Low-complexity components are characterised as standard, low-cost, genericallydefined and backwards compatible. In general, these components are passives and can be procured from multiple suppliers. Examples of this type of component are standard capacitors and resistors.

Essentially, low-complexity is a characteristic of commodity items, whereas highcomplexity is a characteristic of tailored items. Therefore, the difficulty to resolve an obsolescence issue increases with the complexity of the obsolete component, as there are less FFF replacements available.

\section{- Obsolescence Management Levels of Proactiveness}

The third outcome of this study is the classification of the obsolescence management (OM) into five levels of proactiveness based on the mitigation strategies employed, as shown in Figure 5. 


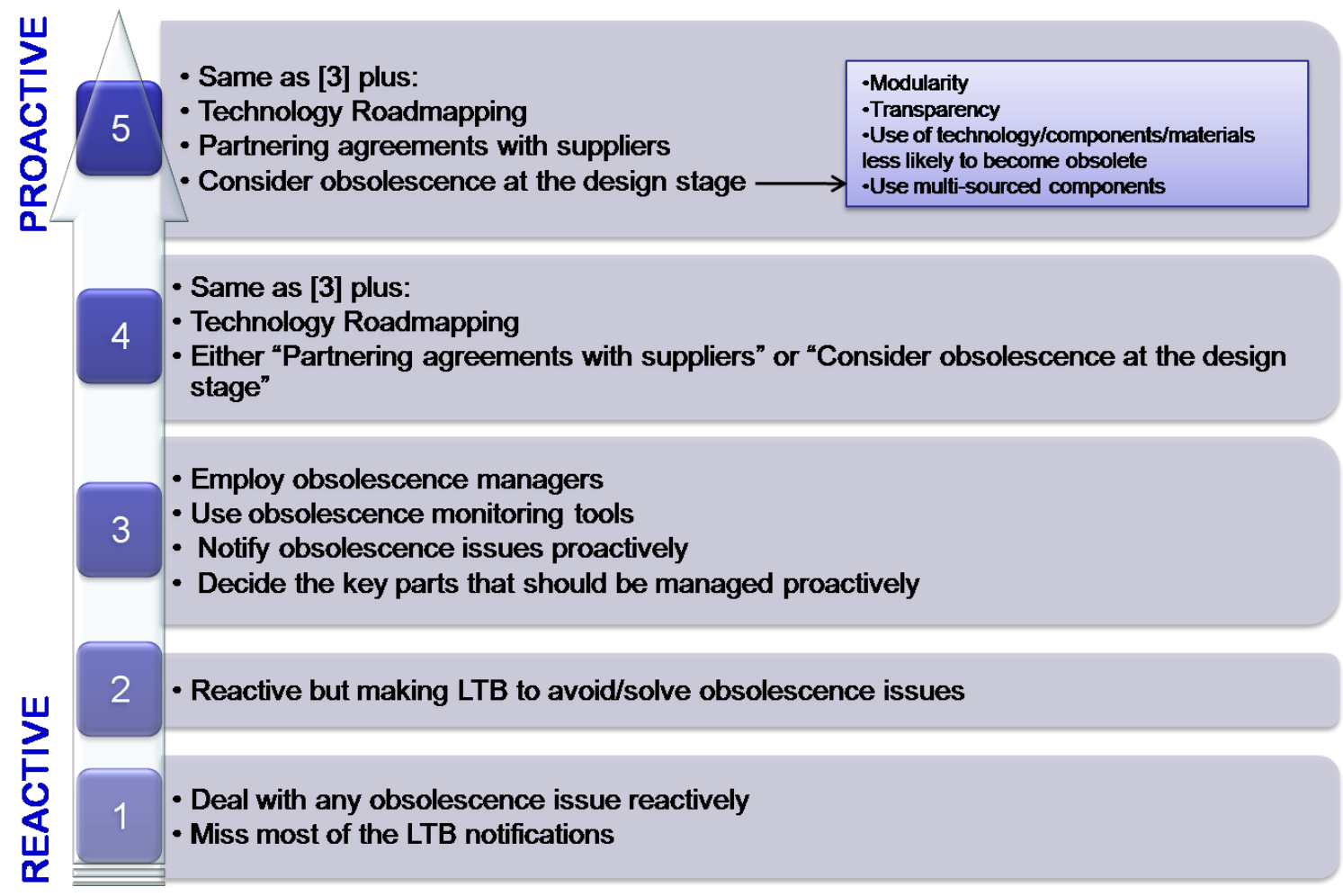

Figure 5 Obsolescence Management Levels

In this classification, level 1 represents a purely reactive strategy whereas level 5 represents the most proactive measures, where all the following proactive strategies are taken:

- Employ obsolescence managers

- Use obsolescence monitoring tools

- Notify obsolescence issues proactively

- Decide which key parts should be managed proactively (after carrying out an obsolescence risk assessment)

- Technology Roadmapping

- Partnering agreements with suppliers

- Consider obsolescence at the design stage:

- Modularity [22]

- Transparency

- Use of technology, components and materials that are less likely to become obsolete

- Use multi-sourced components 
The more mitigation strategies are employed, that is to say, the higher the level of proactiveness to manage obsolescence is, the lower the risk of obsolescence would be. The risk is reduced twofold; the probability of having an obsolescence issue diminishes and the impact of the potential obsolescence issue decreases as well.

\subsection{Results in Phase 1}

A comparison between the outcomes of the first and second round of the Delphi study indicates that most of the figures received little adjustment during the second phase (average of $\pm 1.43 \%$ ). However, some figures changed in $7 \%$ or more, such as the probability of using cannibalisation when the obsolescence management level is 1 (totally reactive) to resolve an obsolescence issue for any level of complexity. As shown in Table 4, the cannibalisation probability decreased from $38.3 \%$ to $24.8 \%$ for low-complexity components, from $35.1 \%$ to $26.7 \%$ for medium-complexity components and from $32.1 \%$ to $24.2 \%$ for high-complexity components. For lowcomplexity components, the probability of using a FFF replacement increased from $7.9 \%$ to $18.6 \%$ when the obsolescence management level is 1 and from $22.9 \%$ to $29.9 \%$ when the obsolescence management level is 4 . The reason for this adjustment is that the figures resulting from the first round of the Delphi study for this case were higher than reality for cannibalisation and lower for FFF replacement.

Table 4 Comparison between Results from First and Second Rounds of Delphi Study

\begin{tabular}{|c|c|c|c|c|c|}
\hline OM Level & $\begin{array}{c}\text { Complexity } \\
\text { Level }\end{array}$ & $\begin{array}{c}\text { Resolution } \\
\text { Approach }\end{array}$ & Round 1 & Round 2 & Difference \\
\hline 1 & Low & Cannibalisation & $38.3 \%$ & $24.8 \%$ & $-13.6 \%$ \\
\hline 1 & Medium & Cannibalisation & $35.1 \%$ & $26.7 \%$ & $-8.4 \%$ \\
\hline 1 & High & Cannibalisation & $32.1 \%$ & $24.2 \%$ & $7.9 \%$ \\
\hline 1 & Low & FFF Replacement & $7.9 \%$ & $18.6 \%$ & $10.7 \%$ \\
\hline 4 & Low & FFF Replacement & $22.9 \%$ & $29.9 \%$ & $7.0 \%$ \\
\hline
\end{tabular}

This comparison of results between the first and second rounds shows that the differences are minor and, hence, no further iterations in the Delphi method are required. 


\subsection{Results in Phase 2}

As a result of the "Obsolescence Resolution Workshop", the "FFF replacement" resolution approach was broken down into "Equivalent" and "Alternative". The results (Table 5) reflect that for all level of proactiveness in managing obsolescence, the probability of finding an equivalent instead of an alternative is higher for lowcomplexity components, but lower for medium and high-complexity components. This is coherent with the characteristics defined for each level of complexity: the higher the complexity of the obsolete component is, the more difficult to find an equivalent to replace it would be.

Table 5 Results of Dividing FFF Replacement into Equivalent and Alternative

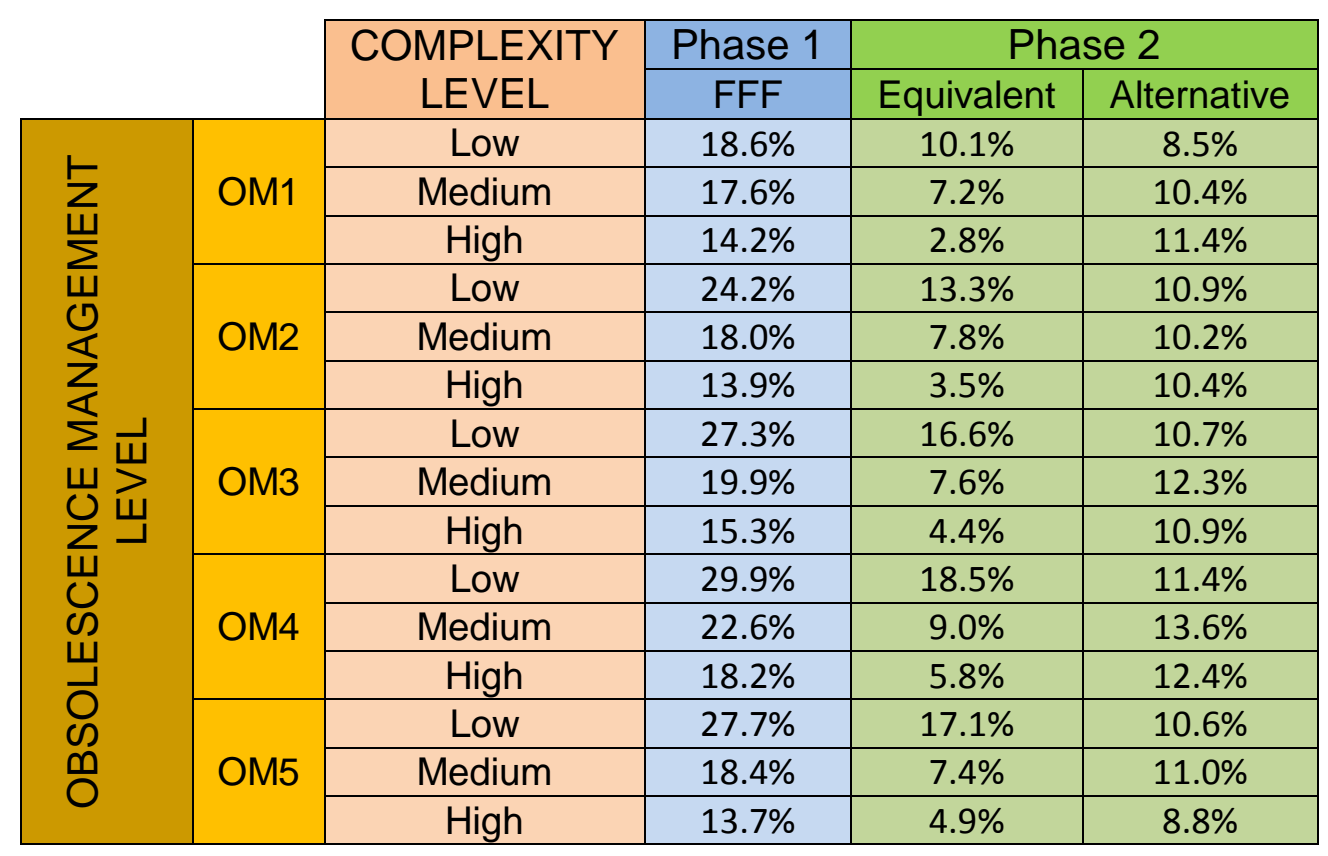




\subsection{Results in Phase 3}

Figure 6 illustrates the probabilities of using each resolution approach have varied after taking into account the theoretical trends conceived by experts. It can be appreciated that less than $35 \%$ of those values have been modified in more that $2 \%$, and none has been modified in more than $9.5 \%$. This indicates that the adjustments made to adapt the ORPs to follow the theoretical trends brought about necessary but not substantial changes to the figures.

\begin{tabular}{|l|c|c|c|c|c|}
\cline { 2 - 7 } \multicolumn{1}{c|}{} & \multicolumn{5}{c|}{ OBSOLESCENCE MANAGEMENT LEVEL } \\
\hline \multicolumn{1}{c|}{ LOW COMPLEXITY } & OM1 & OM2 & OM3 & OM4 & OM5 \\
\hline Existing Stock & $1.4 \%$ & $-2.3 \%$ & $-6.8 \%$ & $-5.5 \%$ & $-1.7 \%$ \\
\hline LTB & $0.9 \%$ & $-1.6 \%$ & $-0.2 \%$ & $-1.0 \%$ & $-3.9 \%$ \\
\hline Cannibalisation & $1.6 \%$ & $-0.1 \%$ & $0.0 \%$ & $-0.2 \%$ & $-0.6 \%$ \\
\hline Equivalent & $0.6 \%$ & $-1.2 \%$ & $-0.1 \%$ & $-1.0 \%$ & $-2.9 \%$ \\
\hline Alternative & $0.5 \%$ & $-1.0 \%$ & $-0.1 \%$ & $-0.6 \%$ & $-1.9 \%$ \\
\hline Authorised Aftermarket & $-6.3 \%$ & $-1.0 \%$ & $-0.1 \%$ & $-0.8 \%$ & $-0.9 \%$ \\
\hline Emulation & $0.0 \%$ & $1.5 \%$ & $-0.5 \%$ & $0.8 \%$ & $1.6 \%$ \\
\hline Minor Redesign & $0.5 \%$ & $6.1 \%$ & $7.8 \%$ & $6.4 \%$ & $5.3 \%$ \\
\hline Major Redesign & $0.8 \%$ & $-0.3 \%$ & $0.0 \%$ & $1.9 \%$ & $4.9 \%$ \\
\hline
\end{tabular}

\begin{tabular}{|l|c|c|c|c|c|}
\cline { 2 - 6 } \multicolumn{1}{c|}{} & \multicolumn{5}{c|}{ OBSOLESCENCE MANAGEMENT LEVEL } \\
\hline MEDIUM COMPLEXITY & OM1 & OM2 & OM3 & OM4 & OM5 \\
\hline Existing Stock & $0.8 \%$ & $-4.1 \%$ & $-1.7 \%$ & $-2.9 \%$ & $-3.7 \%$ \\
\hline LTB & $0.6 \%$ & $-3.0 \%$ & $-1.8 \%$ & $-3.7 \%$ & $-3.9 \%$ \\
\hline Cannibalisation & $1.2 \%$ & $-0.3 \%$ & $0.0 \%$ & $-0.3 \%$ & $3.6 \%$ \\
\hline Equivalent & $0.3 \%$ & $-1.3 \%$ & $-0.5 \%$ & $-2.0 \%$ & $-4.6 \%$ \\
\hline Alternative & $0.5 \%$ & $-1.7 \%$ & $-0.9 \%$ & $-0.4 \%$ & $-3.9 \%$ \\
\hline Authorised Aftermarket & $-3.5 \%$ & $2.9 \%$ & $2.6 \%$ & $0.7 \%$ & $-0.5 \%$ \\
\hline Emulation & $-1.0 \%$ & $1.7 \%$ & $-0.1 \%$ & $2.5 \%$ & $1.1 \%$ \\
\hline Minor Redesign & $0.4 \%$ & $7.0 \%$ & $2.8 \%$ & $4.3 \%$ & $7.2 \%$ \\
\hline Major Redesign & $0.6 \%$ & $-1.3 \%$ & $-0.4 \%$ & $1.8 \%$ & $4.6 \%$ \\
\hline
\end{tabular}

\begin{tabular}{|l|c|c|c|c|c|}
\cline { 2 - 7 } \multicolumn{1}{c|}{} & \multicolumn{5}{c|}{ OBSOLESCENCE MANAGEMENT LEVEL } \\
\hline \multicolumn{1}{c|}{ HIGH COMPLEXITY } & OM1 & OM2 & OM3 & OM4 & OM5 \\
\hline Existing Stock & $0.5 \%$ & $-3.1 \%$ & $-3.1 \%$ & $2.8 \%$ & $-1.8 \%$ \\
\hline LTB & $0.7 \%$ & $-3.1 \%$ & $-3.7 \%$ & $-8.7 \%$ & $-2.9 \%$ \\
\hline Cannibalisation & $0.8 \%$ & $-0.2 \%$ & $-0.1 \%$ & $3.9 \%$ & $3.7 \%$ \\
\hline Equivalent & $0.8 \%$ & $0.5 \%$ & $0.4 \%$ & $0.3 \%$ & $-1.6 \%$ \\
\hline Alternative & $0.4 \%$ & $-1.4 \%$ & $-1.6 \%$ & $-2.1 \%$ & $-7.2 \%$ \\
\hline Authorised Aftermarket & $-2.0 \%$ & $9.5 \%$ & $7.4 \%$ & $-1.5 \%$ & $-1.3 \%$ \\
\hline Emulation & $-2.0 \%$ & $-0.5 \%$ & $1.7 \%$ & $0.6 \%$ & $-0.4 \%$ \\
\hline Minor Redesign & $0.4 \%$ & $0.8 \%$ & $-2.7 \%$ & $3.2 \%$ & $7.0 \%$ \\
\hline Major Redesign & $0.4 \%$ & $-2.4 \%$ & $1.6 \%$ & $1.5 \%$ & $4.5 \%$ \\
\hline
\end{tabular}

The values for which the variation is higher than $2 \%$ have been highlighted

Figure 6 Comparison Between ORP Before and After Trends Refinement 
Two different trends were analysed for each resolution approach. The first trend represents the evolution of the probability for each level of complexity across the different levels of proactiveness to manage obsolescence. An example of this analysis is provided in Figure 7. The second trend represents the evolution of the probability for each level of proactiveness across the different levels of complexity in the obsolete component. An example of this analysis is provided in Figure 8.

Before Refining

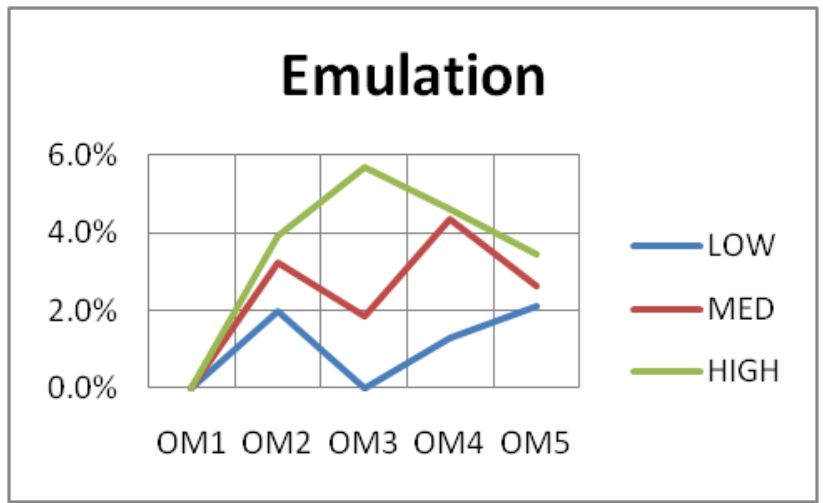

After Refining

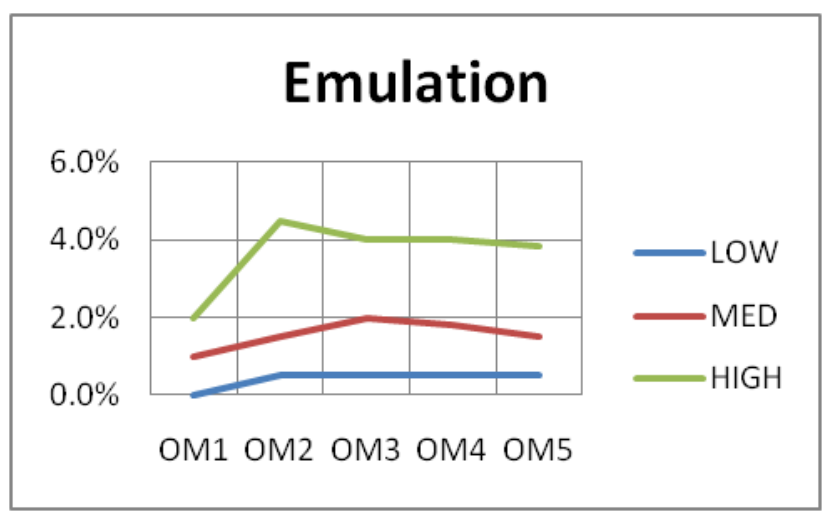

Figure 7 Emulation Probability Trends across Level of Proactiveness for OM Before and After Trends Refinement

According to the experts, for low and medium complexity components, the probability of using emulation is expected to be low and flat across the different levels of OM because this resolution approach is very expensive to be applied to that type of components (Figure 7). The trend for high complexity components is expected to become flat from OM level 3 to 5 . Additionally, the fact that, for every level of proactiveness in obsolescence management, the probability of using emulation is higher for high-complexity components and lower for low-complexity components is coherent with the theoretical expectations. 
Before Refining

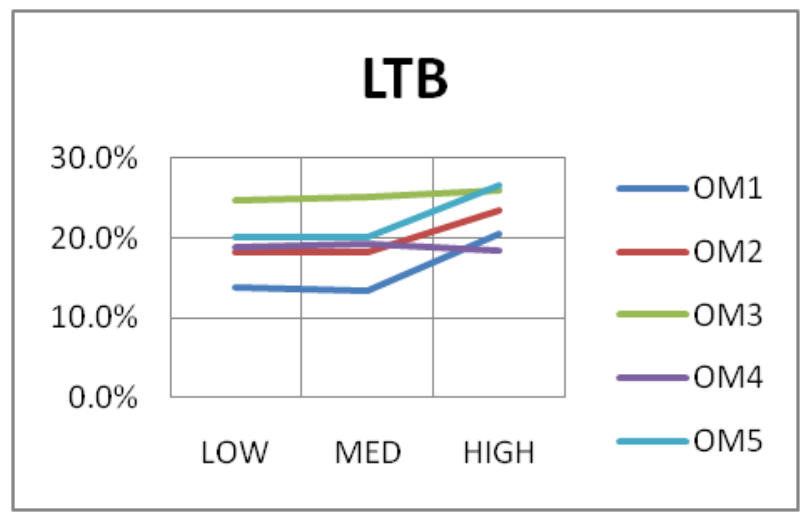

After Refining

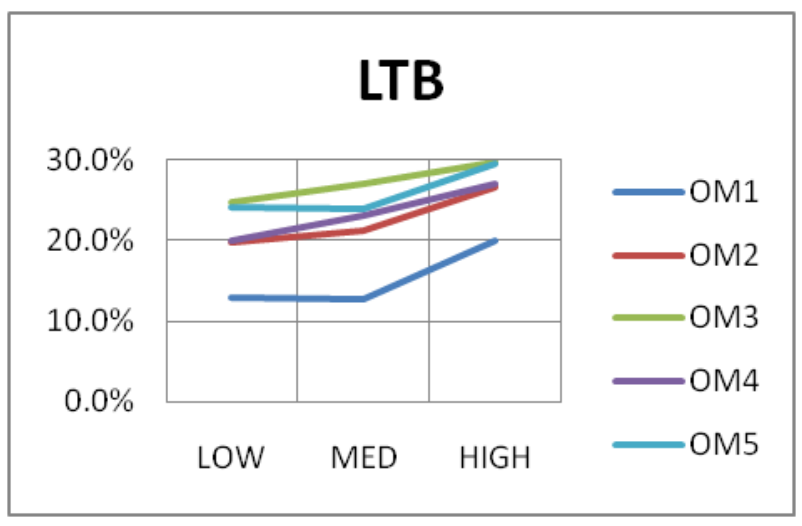

Figure 8 Last-Time Buy Probability Trends across Level of Complexity Before and After Trends Refinement

The trend for all OM levels should be increasing because it is more likely to make a Last-Time Buy (LTB) for high complexity components in order to avoid redesigns. Therefore, the trend for OM level 4 has been adapted to fit the theoretical trend (Figure 8). Additionally, it is logical that for the most reactive level of obsolescence management (OM1) the probability of making a last time buy (LTB) is lower that for more proactive strategies, because most of the product change notifications (PCN) will be ignored or not addressed on time. Therefore, most of the LTBs will be missed.

\section{- Obsolescence Resolution Profiles (ORPs)}

Finally, the main outcomes of this study are the obsolescence resolution profiles (Figure 9). It shows a total of 15 columns (ORPs), where each one represents the probability of using each obsolescence resolution approach to tackle an obsolescence issue. Each obsolescence resolution profile is characterised by one level of component complexity (low, medium or high) and one level of proactiveness for obsolescence management (from 1 to 5 , where 1 represents total reactiveness and 5 represents the highest level of proactiveness). For instance, if a low-complexity component becomes obsolete and obsolescence is managed at the lowest level of proactiveness (OM1), then the probability of making a Last Time Buy (LTB) is 12.9\% and the probability of solving the obsolescence issue by doing a minor redesign is $7.2 \%$. 


\begin{tabular}{|l|c|c|c|c|c|}
\cline { 2 - 6 } \multicolumn{1}{c|}{} & \multicolumn{4}{c|}{ OBSOLESCENCE MANAGEMENT LEVEL } \\
\hline \multicolumn{1}{c|}{ LOW COMPLEXITY } & OM1 & OM2 & OM3 & OM4 & OM5 \\
\hline Existing Stock & $19.7 \%$ & $28.8 \%$ & $25.0 \%$ & $24.4 \%$ & $20.0 \%$ \\
\hline LTB & $12.9 \%$ & $19.9 \%$ & $24.8 \%$ & $19.9 \%$ & $24.1 \%$ \\
\hline Cannibalisation & $23.2 \%$ & $1.4 \%$ & $0.5 \%$ & $3.1 \%$ & $3.4 \%$ \\
\hline Equivalent & $9.42 \%$ & $14.5 \%$ & $16.7 \%$ & $19.5 \%$ & $20.0 \%$ \\
\hline Alternative & $7.97 \%$ & $11.8 \%$ & $10.8 \%$ & $12.1 \%$ & $12.5 \%$ \\
\hline Authorised Aftermarket & $8.0 \%$ & $12.8 \%$ & $13.1 \%$ & $13.5 \%$ & $14.5 \%$ \\
\hline Emulation & $0.0 \%$ & $0.5 \%$ & $0.5 \%$ & $0.5 \%$ & $0.5 \%$ \\
\hline Minor Redesign & $7.2 \%$ & $6.0 \%$ & $5.0 \%$ & $4.0 \%$ & $3.0 \%$ \\
\hline Major Redesign & $11.6 \%$ & $4.3 \%$ & $3.6 \%$ & $3.0 \%$ & $2.0 \%$ \\
\hline
\end{tabular}

\begin{tabular}{|l|c|c|c|c|c|}
\cline { 2 - 6 } \multicolumn{1}{c|}{} & \multicolumn{5}{c|}{ OBSOLESCENCE MANAGEMENT LEVEL } \\
\hline MEDIUM COMPLEXITY & OM1 & OM2 & OM3 & OM4 & OM5 \\
\hline Existing Stock & $16.1 \%$ & $28.9 \%$ & $25.4 \%$ & $24.6 \%$ & $22.6 \%$ \\
\hline LTB & $12.7 \%$ & $21.3 \%$ & $27.0 \%$ & $23.0 \%$ & $23.9 \%$ \\
\hline Cannibalisation & $25.5 \%$ & $2.2 \%$ & $0.5 \%$ & $2.1 \%$ & $2.0 \%$ \\
\hline Equivalent & $6.9 \%$ & $9.1 \%$ & $8.2 \%$ & $11.0 \%$ & $12.0 \%$ \\
\hline Alternative & $9.9 \%$ & $11.8 \%$ & $13.2 \%$ & $14.0 \%$ & $15.0 \%$ \\
\hline Authorised Aftermarket & $6.0 \%$ & $8.0 \%$ & $10.0 \%$ & $12.0 \%$ & $14.0 \%$ \\
\hline Emulation & $1.0 \%$ & $1.5 \%$ & $2.0 \%$ & $1.8 \%$ & $1.5 \%$ \\
\hline Minor Redesign & $9.3 \%$ & $8.0 \%$ & $7.0 \%$ & $6.0 \%$ & $5.0 \%$ \\
\hline Major Redesign & $12.6 \%$ & $9.3 \%$ & $6.8 \%$ & $5.5 \%$ & $4.0 \%$ \\
\hline
\end{tabular}

\begin{tabular}{|l|c|c|c|c|c|}
\cline { 2 - 6 } \multicolumn{1}{c|}{} & \multicolumn{5}{c|}{ OBSOLESCENCE MANAGEMENT LEVEL } \\
\hline \multicolumn{1}{c|}{ HIGH COMPLEXITY } & OM1 & OM2 & OM3 & OM4 & OM5 \\
\hline Existing Stock & $15.8 \%$ & $26.1 \%$ & $24.6 \%$ & $21.0 \%$ & $18.1 \%$ \\
\hline LTB & $19.9 \%$ & $26.7 \%$ & $29.7 \%$ & $27.0 \%$ & $29.6 \%$ \\
\hline Cannibalisation & $23.4 \%$ & $1.9 \%$ & $0.6 \%$ & $1.0 \%$ & $1.0 \%$ \\
\hline Equivalent & $2.0 \%$ & $3.0 \%$ & $4.0 \%$ & $5.5 \%$ & $6.5 \%$ \\
\hline Alternative & $11.0 \%$ & $11.8 \%$ & $12.5 \%$ & $14.5 \%$ & $16.0 \%$ \\
\hline Authorised Aftermarket & $3.0 \%$ & $5.0 \%$ & $7.0 \%$ & $11.0 \%$ & $13.0 \%$ \\
\hline Emulation & $2.0 \%$ & $4.5 \%$ & $4.0 \%$ & $4.0 \%$ & $3.8 \%$ \\
\hline Minor Redesign & $10.7 \%$ & $10.0 \%$ & $9.0 \%$ & $8.0 \%$ & $6.0 \%$ \\
\hline Major Redesign & $12.2 \%$ & $11.0 \%$ & $8.7 \%$ & $8.0 \%$ & $6.0 \%$ \\
\hline
\end{tabular}

Figure 9 Obsolescence Resolution Profiles

The alternative obsolescence resolution profiles, that represent the likelihood of using each resolution approach during the termination stage of the in-service phase, can be calculated from Figure 9 by reducing by half the probability of using equivalents, alternatives, redesigns and emulations. The existing stock and authorised aftermarket shall remain unchanged, and cannibalisation and Last-time Buy (LTB) should increase proportionally to their probabilities in the original obsolescence resolution profiles. 


\section{DISCUSSION AND CONCLUSIONS}

An overall analysis of the differences between the trend in the ORP resulting from applying the Delphi method and the theoretical trends indicated that the reasons for it may be due to:

- Low sample size. Only 38 experts participated in the first round and 33 in the second round of the Delphi study. In the first round, each expert provided data related to a particular level of obsolescence management (OM), as shown in Table 6. Therefore, it can be argued that the level of uncertainty is high, especially for OM levels 1 and 2 due to the reduced sample size ( 5 and 3 experts). However, the 33 experts that participated on the second round validated the figures for all the OM levels.

Table 6 Number of Experts Participating in the First Round of the Delphi Study for each Obsolescence Management Level

\begin{tabular}{cc}
\hline OBSOLESCENCE MANAGEMENT LEVEL & NUMBER OF EXPERTS \\
\hline OM 1 & 5 \\
OM 2 & 3 \\
OM 3 & 8 \\
OM 4 & 10 \\
OM 5 & 12 \\
\hline
\end{tabular}

- Experts from across the UK defence sector participated in each round of the Delphi Study. Many of them have different backgrounds, and the fact that they work at different levels of the supply chain (e.g. customers, system integrators, manufacturers, suppliers, authorised aftermarket) results in them having different points of view about the resolution of obsolescence issues. For example, the system integrators have a good overall understanding about the likelihood of using each resolution approach because they are usually in charge of managing obsolescence and find solutions for obsolescence issues. However, in many instances the decision on the resolution approach selected relies on the customer, especially when the obsolescence issue becomes expensive to resolve. Suppliers and authorised aftermarket might be aware of the obsolescence related to the components that they are sourcing but do not have access to developments in the rest of the system. 
The point above makes clear the independence across most of the members of the panel for each round of the Delphi Study. Additionally, the fact that most of the experts that participated in the second round (20 out of 33) did not take part in the previous round shows the independence between the experts in the two rounds. Therefore, the potential bias associated with dependencies between experts has been reduced.

The main result of this study is a set of ORPs developed and validated in collaboration with experts in obsolescence from across the UK defence sector. Due to the unavailability of historical data, it was necessary to rely on expert judgement to achieve the results. An adequate number of experts participated in this study, therefore reducing bias coupled with the application of the Delphi method facilitating building consensus. The iterative methodology followed in this study allowed the results to be refined and therefore best represent the actual likelihood of using each resolution approach. An essential finding in this study was the identification of the two major factors that influence the probability of using each resolution approach; the obsolescence management level of proactiveness and the complexity level of the obsolete component.

\section{ACKNOWLEDGEMENTS}

The authors would like to thank the following for assisting their research activities in this area: in particular the industrial collaborators, MoD DE\&S, BAE Systems, GE Aviation, Rolls Royce, Lockheed Martin, Thales Aerospace, Selex Galileo, and specially the Component Obsolescence Group (COG). They also thank the EPSRC and the Cranfield University's Innovative Manufacturing Research Centre (IMRC) for funding the research. This paper is presented as part of the research undertaken by the PSS-COST project at the Decision Engineering Centre (DEC) at Cranfield University. The authors would also like to thank the anonymous reviewers for their insightful and constructive comments on this paper. 


\section{REFERENCES}

[1] Singh, P., Sandborn, P., Lorenson, D. and Geiser, T. Determining optimum redesign plans for avionics based on electronic part obsolescence forecasts. Proceedings of the World Aviation Congress, Phoenix, AZ, SAE International, November 2002.

[2] Pecht, M. G. and Das, D. Electronic part life cycle. IEEE Trans. Comp. Packag. Technol. 2000, 23(1), 190-193. doi:10.1109/6144.833060.

[3] Meyer, A., Pretorius, L. and Pretorius, J. H. C. A model to manage electronic component obsolescence for complex or long life systems. Engineering Management Conference, 2004 Proceedings. IEEE International. 2004, 3(1), 1303-1309. doi:10.1109/ IEMC.2004.14089

[4] Sandborn, P. Designing for technology obsolescence management. Proceedings of the 2007 Industrial Engineering Research Conference, 19-23 May 2007 (Nashville, TN).

[5] Solomon, R., Sandborn, P. and Pecht, M. Electronic part life cycle concepts and obsolescence forecasting. IEEE Transactions on Components and Packaging Technologies. 2000, 23(4), 707-717. doi:10.1109/6144.888857.

[6] Feldman, K. and Sandborn, P. Integrating technology obsolescence considerations into product design planning. Proceedings of the ASME 2007 International Design Engineering Conferences \& Computers and Information in Engineering Conference, Sept. 2007, Las Vegas, NV.

[7] Romero Rojo, F. J., Roy, R. and Shehab, E. Obsolescence management for long-life contracts: state of the art and future trends, International Journal of Advanced Manufacturing Technology (IJAMT). 2009, 49(9-12), 1235-1250. doi: 10.1007/s00170-009-2471-3.

[8] Kerr, C. I. V., Phaal, R. and Probert, D. R. Technology insertion in the defence industry: A primer. Proc. IMechE, Part B: J. Engineering Manufacture. 2008, 222(8), 1009-1023. doi: 10.1243/09544054JEM1080. 
[9] Romero Rojo, F. J., Roy, R., Shehab, E. and Wardle, P. Obsolescence Challenges for Product-Service Systems in Aerospace and defence Industry. The 1st CIRP Industrial Product-Service Systems (IPS2) Conference, 1st-2nd April 2009 (Cranfield University, UK).

[10] Josias, C., Terpenny, J. P. and McLean, K. J. Component obsolescence risk assessment. Proceedings of the 2004 Industrial Engineering Research Conference (IERC), 15-19 May 2004.

[11] Romero Rojo, F. J., Roy, R., Shehab, E., Cheruvu, K. and Mason, P. A Cost Estimating Framework for Electronic, Electrical and Electromechanical (EEE) Components Obsolescence within the Use-Oriented Product-Service Systems Contracts. (Accepted for publication in IMechE part B on $22^{\text {nd }}$ March 2011)

[12] Ministry of defence - component obsolescence resolution cost metrics study, QinetiQ and ARINC, UK, 2004. Available at: http://www.aof.mod.uk/aofcontent/tactical/engineering/downloads/om_resolution _metrics_2004.pdf (accessed 18th October 2010)

[13] Flanagan, J. C. The critical incident technique. Psychological bulletin, July 1954, 51(4), 327-358.

[14] Hollnagel, E. Handbook of cognitive task design, Lawrence Erlbaum, Mahwah (NJ), 2003.

[15] Component Obsolescence Group (COG). www.cog.org.uk (accessed on $10^{\text {th }}$ August 2010).

[16] Skulmoski, G. J., Hartman, F. T. and Krahn, J. The Delphi method for graduate research. Journal of Information Technology Education, 2007, 6(1), 1-21.

[17] Okoli C. and Pawlowski S. D. The Delphi Method as a research tool: An example, design considerations and applications. Information \& Management. 2004, 42(1), 15-29. doi:10.1016/j.im.2003.11.002

[18] Linstone, H. A. and Turoff, M. The Delphi method: techniques and applications, Addison-Wesley, London, 1975. 
[19] Rowe, G. and Wright, G. The Delphi Technique as a Forecasting Tool: Issues and Analysis. International Journal of Forecasting, 1999, 15(4), 353-375.

[20] Keeney, R. L. and von Winterfeldt, D., Probabilities are useful to quantify expert judgments, Reliability Engineering \& System Safety, 1988, 23(4), 293-298. doi:10.1016/0951-8320(88)90041-5.

[21] Robson C. Real World Research: A Resource for Social Scientists and Practitioner-Researchers, Blackwell Publishers Ltd., UK, 2002.

[22] Heilala, J., Montonen, J. and Väätäinen, O. Life cycle and unit-cost analysis for modular reconfigurable flexible light assembly systems, Proceedings of the Institution of Mechanical Engineers, Part B: Journal of Engineering Manufacture, 2008, 222(10), 1289-1299. doi:10.1243/09544054JEM1034. 


\section{APPENDIX 1: QUESTIONNAIRE DELPHI STUDY - ROUND 1}

Aim: To capture the profiles of usage of each resolution approach to resolve obsolescence issues for different types of electronic components.

Name (optional):

Organisation (optional):

Years of Experience:

Question 1. Please evaluate the level of Obsolescence Management applied on your company.

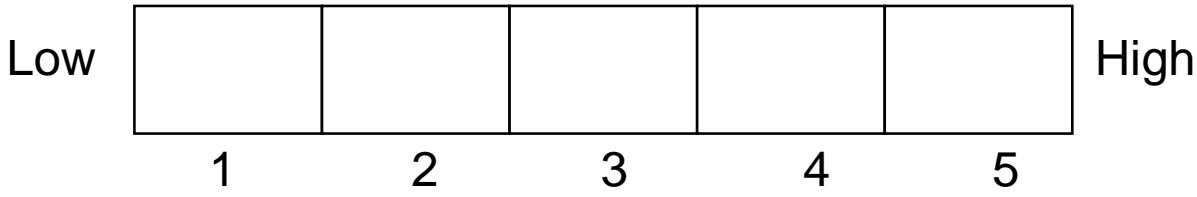

Question 2. Please assess the level of usage of each resolution approach for each level of complexity: (from 0 to 10; 0 represents that the resolution approach is not used at all; 10 represents that the resolution approach is used frequently)

\section{LOW COMPLEXITY}

\begin{tabular}{|c|c|c|c|c|c|c|c|c|c|c|c|}
\hline $\begin{array}{c}\text { RESOLUTION } \\
\text { APPROACH }\end{array}$ & \multicolumn{10}{|c|}{ LEVEL OF USAGE } \\
\hline Existing Stock & $\mathbf{0}$ & $\mathbf{1}$ & $\mathbf{2}$ & $\mathbf{3}$ & $\mathbf{4}$ & $\mathbf{5}$ & $\mathbf{6}$ & $\mathbf{7}$ & $\mathbf{8}$ & $\mathbf{9}$ & $\mathbf{1 0}$ \\
\hline Last Time Buy & $\mathbf{0}$ & $\mathbf{1}$ & $\mathbf{2}$ & $\mathbf{3}$ & $\mathbf{4}$ & $\mathbf{5}$ & $\mathbf{6}$ & $\mathbf{7}$ & $\mathbf{8}$ & $\mathbf{9}$ & $\mathbf{1 0}$ \\
\hline Cannibalisation & $\mathbf{0}$ & $\mathbf{1}$ & $\mathbf{2}$ & $\mathbf{3}$ & $\mathbf{4}$ & $\mathbf{5}$ & $\mathbf{6}$ & $\mathbf{7}$ & $\mathbf{8}$ & $\mathbf{9}$ & $\mathbf{1 0}$ \\
\hline $\begin{array}{c}\text { FFF } \\
\text { Replacement }\end{array}$ & $\mathbf{0}$ & $\mathbf{1}$ & $\mathbf{2}$ & $\mathbf{3}$ & $\mathbf{4}$ & $\mathbf{5}$ & $\mathbf{6}$ & $\mathbf{7}$ & $\mathbf{8}$ & $\mathbf{9}$ & $\mathbf{1 0}$ \\
\hline $\begin{array}{c}\text { Authorised } \\
\text { Aftermarket }\end{array}$ & $\mathbf{0}$ & $\mathbf{1}$ & $\mathbf{2}$ & $\mathbf{3}$ & $\mathbf{4}$ & $\mathbf{5}$ & $\mathbf{6}$ & $\mathbf{7}$ & $\mathbf{8}$ & $\mathbf{9}$ & $\mathbf{1 0}$ \\
\hline $\begin{array}{c}\text { Emulation } \\
\mathbf{0}\end{array}$ & $\mathbf{1}$ & $\mathbf{2}$ & $\mathbf{3}$ & $\mathbf{4}$ & $\mathbf{5}$ & $\mathbf{6}$ & $\mathbf{7}$ & $\mathbf{8}$ & $\mathbf{9}$ & $\mathbf{1 0}$ \\
\hline Minor Redesign & $\mathbf{0}$ & $\mathbf{1}$ & $\mathbf{2}$ & $\mathbf{3}$ & $\mathbf{4}$ & $\mathbf{5}$ & $\mathbf{6}$ & $\mathbf{7}$ & $\mathbf{8}$ & $\mathbf{9}$ & $\mathbf{1 0}$ \\
\hline Major Redesign & $\mathbf{0}$ & $\mathbf{1}$ & $\mathbf{2}$ & $\mathbf{3}$ & $\mathbf{4}$ & $\mathbf{5}$ & $\mathbf{6}$ & $\mathbf{7}$ & $\mathbf{8}$ & $\mathbf{9}$ & $\mathbf{1 0}$ \\
\hline
\end{tabular}




\section{MEDIUM COMPLEXITY}

\begin{tabular}{|c|c|c|c|c|c|c|c|c|c|c|c|}
\hline $\begin{array}{l}\text { RESOLUTION } \\
\text { APPROACH }\end{array}$ & \multicolumn{11}{|c|}{ LEVEL OF USAGE } \\
\hline Existing Stock & 0 & 1 & 2 & 3 & 4 & 5 & 6 & 7 & 8 & 9 & 10 \\
\hline Last Time Buy & 0 & 1 & 2 & 3 & 4 & 5 & 6 & 7 & 8 & 9 & 10 \\
\hline Cannibalisation & 0 & 1 & 2 & 3 & 4 & 5 & 6 & 7 & 8 & 9 & 10 \\
\hline $\begin{array}{c}\text { FFF } \\
\text { Replacement }\end{array}$ & 0 & 1 & 2 & 3 & 4 & 5 & 6 & 7 & 8 & 9 & 10 \\
\hline $\begin{array}{l}\text { Authorised } \\
\text { Aftermarket }\end{array}$ & $\mathbf{0}$ & 1 & 2 & 3 & 4 & 5 & 6 & 7 & 8 & 9 & 10 \\
\hline Emulation & $\mathbf{0}$ & 1 & 2 & 3 & 4 & 5 & 6 & 7 & 8 & 9 & 10 \\
\hline Minor Redesign & $\mathbf{0}$ & 1 & 2 & 3 & 4 & 5 & 6 & 7 & 8 & 9 & 10 \\
\hline Major Redesign & 0 & 1 & 2 & 3 & 4 & 5 & 6 & 7 & 8 & 9 & 10 \\
\hline
\end{tabular}

\section{HIGH COMPLEXITY}

\begin{tabular}{|c|c|c|c|c|c|c|c|c|c|c|c|}
\hline $\begin{array}{c}\text { RESOLUTION } \\
\text { APPROACH }\end{array}$ & \multicolumn{10}{|c|}{ LEVEL OF USAGE } \\
\hline Existing Stock & $\mathbf{0}$ & $\mathbf{1}$ & $\mathbf{2}$ & $\mathbf{3}$ & $\mathbf{4}$ & $\mathbf{5}$ & $\mathbf{6}$ & $\mathbf{7}$ & $\mathbf{8}$ & $\mathbf{9}$ & $\mathbf{1 0}$ \\
\hline Last Time Buy & $\mathbf{0}$ & $\mathbf{1}$ & $\mathbf{2}$ & $\mathbf{3}$ & $\mathbf{4}$ & $\mathbf{5}$ & $\mathbf{6}$ & $\mathbf{7}$ & $\mathbf{8}$ & $\mathbf{9}$ & $\mathbf{1 0}$ \\
\hline Cannibalisation & $\mathbf{0}$ & $\mathbf{1}$ & $\mathbf{2}$ & $\mathbf{3}$ & $\mathbf{4}$ & $\mathbf{5}$ & $\mathbf{6}$ & $\mathbf{7}$ & $\mathbf{8}$ & $\mathbf{9}$ & $\mathbf{1 0}$ \\
\hline $\begin{array}{c}\text { FFF } \\
\text { Replacement }\end{array}$ & $\mathbf{0}$ & $\mathbf{1}$ & $\mathbf{2}$ & $\mathbf{3}$ & $\mathbf{4}$ & $\mathbf{5}$ & $\mathbf{6}$ & $\mathbf{7}$ & $\mathbf{8}$ & $\mathbf{9}$ & $\mathbf{1 0}$ \\
\hline $\begin{array}{c}\text { Authorised } \\
\text { Aftermarket }\end{array}$ & $\mathbf{0}$ & $\mathbf{1}$ & $\mathbf{2}$ & $\mathbf{3}$ & $\mathbf{4}$ & $\mathbf{5}$ & $\mathbf{6}$ & $\mathbf{7}$ & $\mathbf{8}$ & $\mathbf{9}$ & $\mathbf{1 0}$ \\
\hline Emulation & $\mathbf{0}$ & $\mathbf{1}$ & $\mathbf{2}$ & $\mathbf{3}$ & $\mathbf{4}$ & $\mathbf{5}$ & $\mathbf{6}$ & $\mathbf{7}$ & $\mathbf{8}$ & $\mathbf{9}$ & $\mathbf{1 0}$ \\
\hline Minor Redesign & $\mathbf{0}$ & $\mathbf{1}$ & $\mathbf{2}$ & $\mathbf{3}$ & $\mathbf{4}$ & $\mathbf{5}$ & $\mathbf{6}$ & $\mathbf{7}$ & $\mathbf{8}$ & $\mathbf{9}$ & $\mathbf{1 0}$ \\
\hline Major Redesign & $\mathbf{0}$ & $\mathbf{1}$ & $\mathbf{2}$ & $\mathbf{3}$ & $\mathbf{4}$ & $\mathbf{5}$ & $\mathbf{6}$ & $\mathbf{7}$ & $\mathbf{8}$ & $\mathbf{9}$ & $\mathbf{1 0}$ \\
\hline
\end{tabular}




\section{APPENDIX 2: QUESTIONNAIRE DELPHI STUDY - ROUND 2}

\section{OBSOLESCENCE RESOLUTION PROFILES VALIDATION}

Name (optional):

Organisation (optional):

Years of Experience:

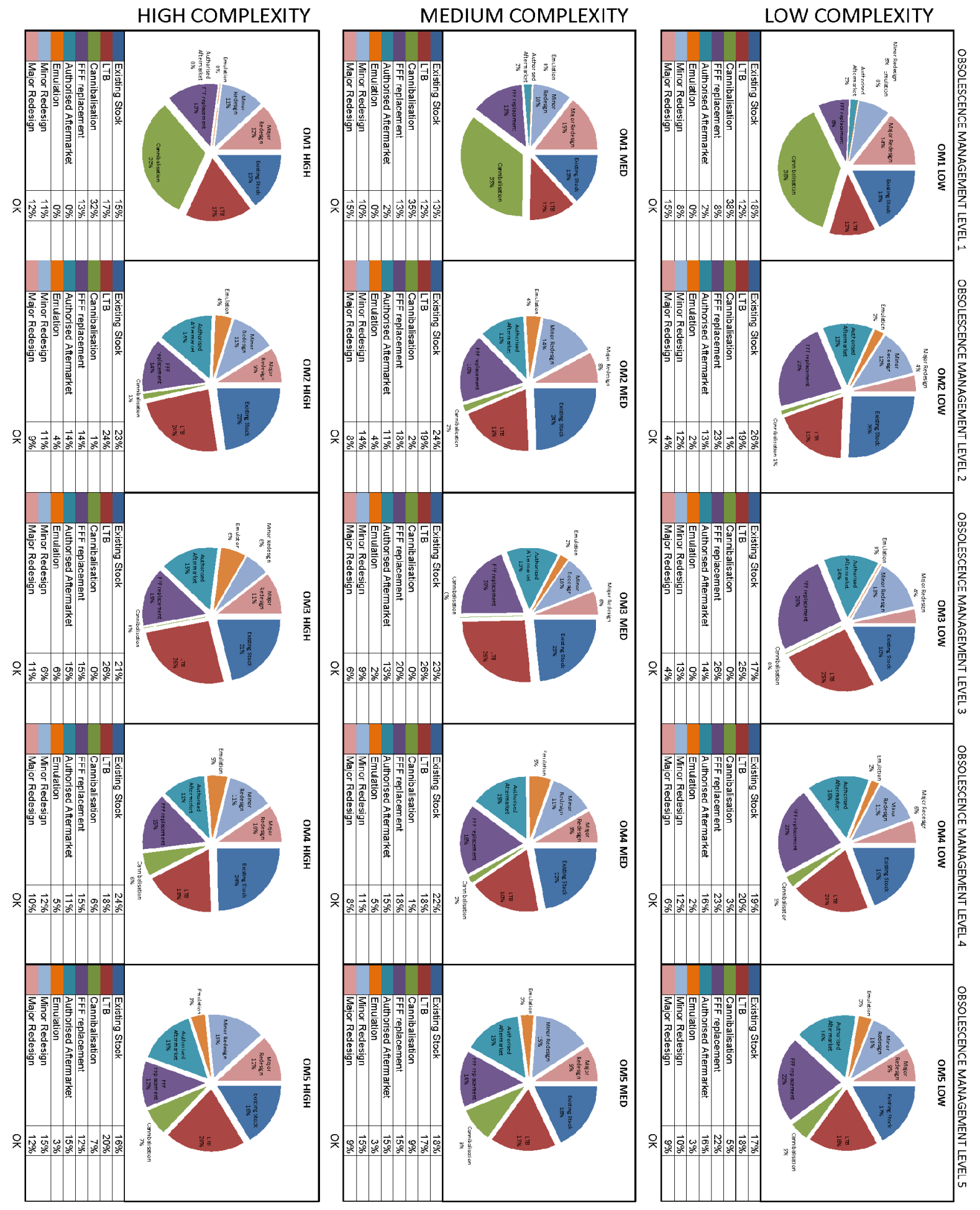

